\title{
Cost of childhood diarrhoea in rural South Africa: exploring cost-effectiveness of universal zinc supplementation
}

\author{
Meera K Chhagan ${ }^{1, *}$, Jan Van den Broeck ${ }^{2}$, Kany-Kany Angelique Luabeya ${ }^{3,4}$, \\ Nontobeko Mpontshane ${ }^{5}$ and Michael L Bennish ${ }^{6}$ \\ 'Division of Maternal and Child Health, Department of Paediatrics, University of KwaZulu-Natal, Durban, P/Bag \\ 7, Congella 4013, South Africa: ${ }^{2}$ Center for International Health, University of Bergen, Bergen, Norway: ${ }^{3}$ South \\ African TB Vaccine Initiative, Institute of Infectious Disease and Molecular Medicine, University of Cape Town, \\ Cape Town, South Africa: ${ }^{4}$ School of Child Health, University of Cape Town, Cape Town, South Africa: ${ }^{5} \mathrm{i}$ \\ Research, Maidenhead, UK: ${ }^{6}$ Mpilonhle, Mtubatuba, South Africa
}

Submitted 3 December 2012: Final revision received 22 June 2013: Accepted 8 July 2013: First published online 12 August 2013

\begin{abstract}
Objective: To describe the cost of diarrhoeal illness in children aged 6-24 months in a rural South African community and to determine the threshold prevalence of stunting at which universal Zn plus vitamin A supplementation (VAZ) would be more cost-effective than vitamin A alone (VA) in preventing diarrhoea.

Design: We conducted a cost analysis using primary and secondary data sources. Using simulations we examined incremental costs of VAZ relative to VA while varying stunting prevalence.

Setting: Data on efficacy and societal costs were largely from a South African trial. Secondary data were from local and international published sources.

Subjects: The trial included children aged 6-24 months. The secondary data sources were a South African health economics survey and the WHO-CHOICE (CHOosing Interventions that are Cost Effective) database.

Results: In the trial, stunted children supplemented with VAZ had $2 \cdot 04$ episodes (95\% CI $1.37,3.05)$ of diarrhoea per child-year compared with 3.92 episodes (95\% CI $3 \cdot 02,5 \cdot 09)$ in the VA arm. Average cost of illness was \$Int $7 \cdot 80$ per episode (10th, 90th centile: \$Int 0·28, \$Int 15.63), assuming a minimum standard of care (oral rehydration and $14 \mathrm{~d}$ of therapeutic $\mathrm{Zn}$ ). In simulation scenarios universal VAZ had low incremental costs or became cost-saving relative to VA when the prevalence of stunting was close to $20 \%$. Incremental cost-effectiveness ratios were sensitive to the cost of intervention and coverage levels.

Conclusions: This simulation suggests that universal VAZ would be cost-effective at current levels of stunting in parts of South Africa. This requires further validation under actual programmatic conditions.
\end{abstract}

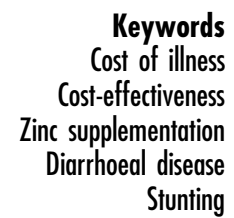

Stunting
Diarrhoeal disease remains, with respiratory disease, among the two main causes of childhood morbidity and mortality in middle- and low-income countries ${ }^{(1)}$. The long-term consequences for growth and cognition are being increasingly recognized as the burden associated with diarrhoeal disease ${ }^{(2)}$. Interventions to reduce diarrhoea burden include the provision of safe water and sanitation, breast-feeding promotion, rotavirus vaccination and therapeutic Zn supplementation during diarrhoea episodes. Prophylactic Zn supplementation has shown efficacy in many trials, but translation of these findings to public health programmes is limited by difficulties in establishing $\mathrm{Zn}$ status and efficacy in different populations and sparse knowledge of feasible strategies for delivery ${ }^{(3)}$.

Zn supplementation for 10-14 d is currently included in standard diarrhoea case management guidelines ${ }^{(4)}$.
Prophylactic $\mathrm{Zn}$ is not currently included in preventive child health programmes. A decision to implement universal Zn supplementation requires knowledge of clinical efficacy, population $\mathrm{Zn}$ status and cost-effectiveness relative to existing programmes that in many developing countries already include routine vitamin A supplementation.

While there are reports on the global effect of prophylactic $\mathrm{Zn}$ on disability-adjusted life years (DALY) ${ }^{(5)}$, policy makers may still find it difficult to translate this information for use in diarrhoea prevention programmes, where efficacy of $\mathrm{Zn}$ supplementation and burden of diarrhoeal illness differ locally. Prevalence of stunting, with initial verification using $\mathrm{Zn}$ intake data, could serve as a crude marker of population $\mathrm{Zn}$ status ${ }^{(6)}$. Knowledge of cost-effectiveness of routine $\mathrm{Zn}$ supplementation at varying prevalences of stunting would therefore be 
informative to planners, for example to plan geographic targeting within a country.

The objectives of the present study were to: (i) describe the cost of diarrhoeal illness in children aged 6-24 months in a rural South African community; and (ii) explore a theoretical threshold prevalence of stunting at which universal Zn plus vitamin A supplementation (VAZ) would be more cost-effective than vitamin A alone (VA). The latter aim arises from the finding in several micronutrient trials, and in a recent meta-analysis, that $\mathrm{Zn}$ supplementation preferentially reduces diarrhoea incidence in stunted children $^{(3,7,8)}$. We maintain universal vitamin A supplementation as a standard of care given that this public health intervention has an evidence base that has already resulted in its general acceptance in child health programmes ${ }^{(9)}$.

\section{Methods}

\section{Sources of information for cost of diarrboeal illness}

The cost of diarrhoeal illness was estimated from three sources of data: (i) a micronutrient trial conducted in rural South Africa (referred to below as KZN trial) ${ }^{(10)}$; (ii) a health service costing survey conducted in urban and rural sites in a South African district by the Health Economics and HIV/AIDS Research Division at the University of KwaZulu-Natal (referred to below as HEARD project) ${ }^{(11,12)}$; and (iii) the WHO CHOosing Interventions that are Cost Effective (CHOICE) project database of the cost of interventions and the costs of in-patient and out-patient visits by region (referred to below as WHO-CHOICE) ${ }^{(13,14)}$. For these international sources that reported costs for base year 2000, the South African Consumer Price Index adjustment factor was used to convert these to year 2004 costs $^{(15)}$. All costs were reported as international dollars (\$Int) with 2004 as the base year. The international dollar accounts for differences in purchasing power across countries and can be converted to domestic currency that would purchase the same quantity of services as $\$ 1$ could purchase in the USA ${ }^{(16)}$.

The KZN trial captured direct and indirect costs borne by families. Data on health service utilization and out-of-pocket costs were collected prospectively from participants. A pre-designed economic questionnaire was completed at weekly home visits conducted for active morbidity surveillance. In the South African public health sector there are no user fee charges for children aged below 5 years. These public health sector care costs were obtained from the HEARD project. The HEARD survey captured direct service provider costs, including consultation time, investigations and drug costs, hospitalization costs, length of stay and referral pattern at different levels of care for different age groups and diagnoses ${ }^{(12)}$.

HEARD data were collected only at public health facilities. KZN trial data were collected within communities and households and captured additional health-seeking behaviours such as consultation with private practitioners or traditional healers and purchase of over-the-counter medications. HEARD described costs from the health provider's perspective, while the KZN trial also captured the burden on families. Both sources captured costs in South African Rand (ZAR). For our analysis we used the exchange rate in 2004 of ZAR 6.88/\$nt 1.

The WHO-CHOICE project estimated average outpatient and hospitalization costs for the Afro-E region using a standardized approach ${ }^{(14)}$. Afro-E, one of fourteen geographic sub-regions defined by global burden of disease estimates, includes countries in sub-Saharan Africa, where HIV/AIDS has a large impact on child (under 5 years) and adult mortality. Hospitalization costs are for public hospitals at $80 \%$ occupancy and include the hotel component of costs. It provides cost per visit for primary-care facilities in this region. It includes capital items but excludes drugs. The latter was obtained from the first two sources.

\section{Calculation of cost per episode of diarrboea}

Direct medical costs included family and health sector costs, and comprised medications, including over-the-counter medication purchases, health workers and traditional healer consultations, primary health-care clinic and hospital outpatient visits, and hospitalization. It included use of public and private sector facilities. Scheduled study visits for data collection were not included as part of the cost of healthseeking. If participants consulted study staff for treatment then a cost equivalent to a visit to a primary health-care facility was assigned.

Non-medical and indirect costs included transport, food purchased at health facilities and cost of child care. Information on productivity losses was estimated from time spent travelling and time lost from work or from school for older siblings, although we are aware of the inherent difficulties in assigning a cost to productivity losses ${ }^{(17)}$. For children who had no health service utilization and zero costs captured for their diarrhoea episodes, we examined the influence of assigning a basic cost for each diarrhoea episode. This basic cost included cost of two weeks of $\mathrm{Zn}$ supplementation according to current treatment guidelines and two packets of oral rehydration powder. The rationale for allocating these basic costs was to avoid underestimating costs of illness simply because a basic standard of care was not available or accessible to all children with diarrhoea. The current restructuring of the community health worker programme in South Africa is already incorporating these interventions for wide scale-up.

Combining the information from these sources, we estimated the cost per episode as total direct costs plus total indirect costs averaged over the number of diarrhoea episodes for which cost information was collected, including those episodes that had zero cost recorded. The cost of illness analysis was conducted from a societal perspective; that is, costs were included irrespective of who was paying. We calculated the arithmetic mean as 
well as the bootstrap mean and standard error of the mean derived from 1000 bootstrap replications because of the skewed nature of the cost data.

In assigning monetary values to productivity losses, we used the minimum wage in South Africa in 2004 to assign a cost for time missed from work during a child's diarrhoea episode.

\section{Cost of supplementation}

We referred to WHO-CHOICE for cost of universal VA supplementation $^{(5)}$. These programme-level costs are meant to include all resources required to establish and maintain the intervention, and include those of administration, publicity, training and delivery of the intervention. To date there is sparse information available on programmatic costs of preventive $\mathrm{Zn}$ supplementation. For preventive $\mathrm{Zn}$ supplementation we therefore used published sources of costing information for micronutrient powders ${ }^{(18)}$. The costs described in these sources applied to $95 \%$ and $100 \%$ coverage, respectively. We estimated that $80 \%$ coverage would cost about 30\% lower than $95 \%$ coverage $^{(18)}$.

\section{Efficacy of supplementation}

For base case scenarios, measures of the effect on incidence of diarrhoea were obtained from the KZN trial. The trial found that stunted children had fewer diarrhoea episodes if supplemented with VAZ compared with VA ${ }^{(7)}$. There were no detectable differences in non-stunted children. Since this trial enrolled too few HIV-infected children to detect meaningful differences for this group, we instead used published information on diarrhoeal reduction among $\mathrm{HIV}$-infected children from another $\mathrm{Zn}$ supplementation safety trial conducted in South Africa ${ }^{(19)}$. We also examined the possibility of $\mathrm{Zn}$ preventing diarrhoea among both stunted and non-stunted children, although with smaller effect in the latter, as described in other international studies. Last, we explored a scenario where stunting prevalence was not considered but diarrhoea reduction with VAZ was assumed to be that described in a recently published meta-analysis ${ }^{(3)}$.

\section{Analytic approach}

The measure of cost-effectiveness used in the present analysis is the incremental cost-effectiveness ratio (ICER) of universal VAZ given to all infants starting at age 12 months compared with VA. This is expressed as cost per additional episode of diarrhoea prevented. The following equation was used for ICER:

$$
\mathrm{ICER}=\frac{\text { Net } \text { cost }_{\mathrm{VAZ}}}{\text { Cases prevented }} \text { pAZ }_{\mathrm{VAZ}},
$$

where

$$
\begin{aligned}
\text { Net } \text { cost }_{\mathrm{VAZ}}= & (\text { Intervention cost } \\
& -\left(\text { Intervention } \text { cost }_{\mathrm{VA}}+\text { Illness cost }_{\mathrm{VAZ}}\right) \\
& \text { Illness cost } \left.\mathrm{VA}_{\mathrm{VA}}\right) .
\end{aligned}
$$

Net cost is the difference in costs of interventions minus the cost of averted disease ${ }^{(20)}$. Negative net costs generally imply savings and support implementation of such interventions. If the intervention were to target stunted children only, then the cost involved in identifying stunted children would need to be included. If the intervention is given universally, but the effect size differs in subgroups of stunted and non-stunted children, then the net effect would depend on the proportionate size of the subgroups. If the intervention was preventive only among stunted children, then the net cost and cases prevented would apply only to stunted children. A dominant intervention would be one that reduces diarrhoea (positive denominator) at a net cost saving (negative numerator). An intervention that reduces diarrhoea at a cost (positive numerator) needs further evaluation of whether the cost of achieving a unit benefit is justified. This usually involves comparison of the ICER with that of other interventions, which requires scaling them to a common denominator such as DALY. The Commission on Macroeconomics and Health recommends that an intervention be considered highly cost-effective if it costs less than the Gross National Income per capita $^{(21)}$. Various cut-offs for cost-effectiveness are used however and these may be highly sensitive to different local and regional factors ${ }^{(22)}$.

Since the preventive effect of VAZ on diarrhoea incidence was observed only among stunted children in the South African trial, we conducted a threshold analysis to determine the theoretical prevalence of stunting at which this intervention would be more cost-effective than VA in preventing diarrhoea. We simulated a hypothetical cohort of 10000 children aged 12 months supplemented with VA or VAZ for a year. This is based on meta-analyses that showed greatest benefit in terms of diarrhoea prevention accruing after 12 months with $\mathrm{Zn}$ supplementation ${ }^{(3)}$. The model included information on effectiveness of $\mathrm{Zn}$ plus vitamin A supplementation, cost of diarrhoea episodes and cost of universal supplementation at varying levels of coverage. We calculated ICER for a range of stunting prevalence to assess a level at which universal VAZ would be most cost-effective in preventing diarrhoeal disease assuming that this protective effect is limited to stunted children. All costs were adjusted to base year 2004 for these analyses. We projected all costs over one year and so did not include a discounting rate. We used Microsoft ${ }^{\circledR}$ Office Excel 2010 software for this simulation.

Our main assumptions were: (i) initial coverage target of $80 \%$; (ii) instead of zero costs, the minimum cost for diarrhoea episodes would be that of two weeks' supply of $\mathrm{Zn}$ and two oral rehydration sachets; (iii) efficacy of interventions vary in direct proportion to coverage, hence we adjusted magnitude of effect for varying coverage levels; and (iv) cost of increasing coverage is anticipated to be $30 \%$ greater for increasing coverage from 80 to $95 \%{ }^{(18)}$.

We varied several key parameters to examine how robust the threshold analysis was to varying conditions. 
We performed one-way and multi-way sensitivity analyses varying intervention cost, coverage and cost of illness. We varied magnitude of efficacy using the lower and upper $95 \%$ confidence interval for efficacy from the trial results. We examined scenarios where benefit occurred in both stunted and non-stunted children, with lower effect size in the latter ${ }^{(8)}$. We explored doubling the cost of $\mathrm{Zn}$ to cater for higher distribution costs. We explored a scenario with $5 \%$ prevalence of HIV among infants, which will be realistic in the South African setting. Lastly, we examined ICER if stunting prevalence was not considered but estimates of diarrhoea reduction reported in meta-analyses were applied to the baseline observed in the South African trial ${ }^{(3)}$.

\section{Etbical review}

The Biomedical Research Ethics Committee of the University of KwaZulu-Natal and the Institutional Review Board of Tufts-New England Medical Center approved the KZN trial (ClinicalTrials.gov identifier: NCT00156832). The study was conducted according to the guidelines laid down in the Declaration of Helsinki. Written informed consent was provided by a parent or guardian for all participating children. The HEARD study had approval from the Biomedical Research Ethics Committee, University of KwaZulu-Natal, plus permission from local health authorities and facilities included in the study sample.

\section{Results}

Information on health-seeking and costs was available for 318 children contributing 1563 episodes of diarrhoea during the micronutrient trial. For 959/1563 episodes there was no health service utilization and zero cost recorded. The main categories of health care are shown in Table 1 . Only $9 / 318$ children had access to private health insurance. Of these nine, four consulted the public sector services. Caregivers missed work for twenty-three episodes of diarrhoea amounting to a total of $57 \mathrm{~d}$, while older siblings missed school for seven episodes amounting to a total of $16 \mathrm{~d}$.

\section{Estimates of costs of illness}

The average cost per diarrhoeal episode was \$Int 7.34 (Table 2) after assigning a basic cost to reflect standard care for those episodes with zero costs and no health service utilization. Due to many episodes with no healthseeking the cost per episode was highly skewed, with the 10th and 90th centile being \$Int $0 \cdot 28$ and \$Int $15 \cdot 63$ per episode, respectively. Of the total cost, $65 \%$ was borne by service providers and $35 \%$ by families. Direct costs comprised $91 \%$ of all costs. Doctor consultations and hospitalization contributed most to costs. The average cost per episode was $\$$ Int 6.92 if we did not assign a basic cost to reflect standard of care, with the 10th and 90th centile being $\$$ Int $0 \cdot 00$ and $\$$ Int $15 \cdot 35$, respectively. If we assigned a basic cost plus a monetary value to productivity losses for days lost from work, the average cost increased to $\$$ Int $7 \cdot 80 /$ episode.

\section{Estimates of cost of supplementation}

Using WHO estimates and after adjusting to base year 2004 Consumer Price Index, we estimated at 80\% coverage a cost for 10000 children of \$Int 37277 for VA supplementation alone ${ }^{(5)}$; and using cost of micronutrient powders from the World Bank Report, \$Int 62037 for $\mathrm{VAZ}^{(18)}$. At 95\% coverage the cost would be $\$$ Int 67100 and $\$$ Int 111668 , respectively. If we doubled the cost of Zn supplementation, then VAZ would cost \$Int 82960 and \$nt 149328 at $80 \%$ and $95 \%$ coverage, respectively.

Table 2 Cost of diarrhoeal illness among 318 children aged 6-24 months from societal perspective

\begin{tabular}{lc}
\hline Category of cost & Cost (\$Int) \\
\hline $\begin{array}{l}\text { Direct costs (imputing standard of care for all } \\
\text { episodes with zero costs) }\end{array}$ & 10431 \\
$\begin{array}{l}\text { Indirect costs (reported cost of food, travel and paid } \\
\quad \text { child care) }\end{array}$ & 1041 \\
Total direct plus indirect & 11473 \\
Mean cost per episode (11473/1563) & $7 \cdot 34$ \\
Mean cost per episode including productivity losses & $7 \cdot 80$ \\
Bootstrap & $7 \cdot 12$ \\
Mean & $0 \cdot 68$ \\
SE & \\
\hline
\end{tabular}

Table 1 Cost of health services for 1563 episodes of diarrhoea among 318 children aged 6-24 months

\begin{tabular}{lcrr}
\hline Service utilized & No. of children & No. of episodes of diarrhoea* & Total cost (\$lnt) $^{*}$ \\
\hline Out-patient visit to private doctor & 80 & 103 & 1140 \\
Out-patient visit to clinic & 144 & 209 & 332 \\
Out-patient visit to hospital & 190 & 26 & 1221 \\
Visit to traditional healer & 20 & 23 & 255 \\
Admission to public hospital & 21 & 32 & 2605 \\
Visit or admission to private hospital & 9 & 110 & 1363 \\
Over-the-counter medications & 65 & 138 & 261 \\
Consulted study staff & 93 & 234 & 4 \\
Actual use of ORS & 129 & 1563 & 55 \\
Imputed standard of care: short course Zn and 2 packs of ORS & 318 & 437 \\
\hline
\end{tabular}

ORS, oral rehydration salts.

*More than one service was used for individual episodes of diarrhoea. 


\section{Estimates of efficacy of Zn supplementation}

In the KZN trial stunted children supplemented with VAZ had 2.04 episodes (95\% CI 1.37, 3.05) of diarrhoea per year compared with 3.92 episodes (95\% CI 3.02, 5.09) per year in children who received VA. The difference between non-stunted groups was not statistically significant, so in our base case we included reduction among stunted children only and zero effect among nonstunted. Alternative scenarios included reduction of $1 \cdot 2$ episodes of diarrhoea per child per year among stunted children and 0.36 episodes per child per year among non-stunted children as described by Umeta et $a l .{ }^{(8)}$. For calculation of ICER without geographic targeting by stunting prevalence, we used relative risk of diarrhoea incidence among the VAZ group of $0 \cdot 80$ compared with
$\mathrm{VA}^{(3)}$, with the latter having $2 \cdot 92$ episodes per child per year based on the KZN trial.

\section{Incremental cost-effectiveness ratios and threshold analysis}

For our base scenario we calculated ICER at $80 \%$ coverage and $15 \%$ prevalence of stunting (Table 3). At 15\% prevalence of stunting VAZ had a relatively low incremental cost of $\$$ Int 1.23 per additional case of diarrhoea prevented relative to VA alone. In the threshold analysis, keeping other base case conditions constant, VAZ became cost-saving when stunting prevalence was close to $20 \%$ (Table 4). We varied several parameters in our sensitivity analyses, some of these are presented in Table 5. With increasing stunting prevalence, routine $\mathrm{Zn}$ supplementation

Table 3 Calculation of incremental cost-effectiveness ratio (ICER) assuming $80 \%$ coverage*

\begin{tabular}{|c|c|c|c|c|}
\hline Row & Variable & Base case & Data source & Range for sensitivity analysis \\
\hline a1 & Prevalence of stunting & $15 \%$ & Hypothetical & $5-50 \%$ \\
\hline a2 & Population size & 10000 & Hypothetical & \\
\hline a3 & No. of stunted children & 1500 & $\mathrm{a} 1 \times \mathrm{a} 2$ & \\
\hline a4 & No. of non-stunted children & 8500 & a2 $\times(1-a 1)$ & \\
\hline b1 & Cost of illness per case & $\$ 7 \cdot 80$ & KZN trial, sources ${ }^{(12-14)}$ & $\begin{array}{l}\$ 0 \cdot 28, \$ 7 \cdot 34, \$ 7 \cdot 80 \\
\text { (note base case includes } \\
\text { productivity losses) }\end{array}$ \\
\hline b2 & Cost of VA at $80 \%$ coverage & $\$ 37277$ & Source ${ }^{(5)}$ & $\$ 67100$ for $95 \%$ coverage \\
\hline b3 & Cost VAZ at $80 \%$ coverage & $\$ 62037$ & Source ${ }^{(18)}$ & $\$ 111668$ for $95 \%$ coverage \\
\hline \multicolumn{5}{|l|}{ Base case (VA only) } \\
\hline c1 & $\begin{array}{l}\text { Incidence of diarrhoea in stunted } \\
\text { children }\end{array}$ & 3.92 & $\mathrm{KZN}$ trial & $3 \cdot 02,5 \cdot 09$ \\
\hline $\mathrm{c} 2$ & $\begin{array}{l}\text { No. of episodes in stunted } \\
\text { children }\end{array}$ & 5880 & $c 1 \times a 3$ & \\
\hline c3 & Cost of illness & $\$ 45864$ & $\mathrm{~b} 1 \times \mathrm{c} 2$ & \\
\hline c4 & Total cost with VA & $\$ 83141$ & $\mathrm{c} 3+\mathrm{b} 2$ & \\
\hline \multicolumn{5}{|l|}{ Alternative (VAZ) } \\
\hline d1 & $\begin{array}{l}\text { Incidence of diarrhoea in stunted } \\
\text { children }\end{array}$ & $2 \cdot 04$ & $\mathrm{KZN}$ trial & $1 \cdot 37,3 \cdot 05$ \\
\hline $\mathrm{d} 2$ & $\begin{array}{l}\text { No. of episodes in stunted } \\
\text { children }\end{array}$ & 3060 & d1 $\times$ a3 & \\
\hline d3 & Cost of illness & $\$ 23868$ & $\mathrm{~b} 1 \times \mathrm{d} 2$ & \\
\hline d4 & Total cost with VAZ & $\$ 85905$ & $d 3+b 3$ & \\
\hline \multicolumn{5}{|l|}{ Increment } \\
\hline e1 & $\begin{array}{l}\text { Cases prevented } \\
\qquad(=\text { denominator for ICER) }\end{array}$ & 2256 & $\begin{array}{l}1.88 \times \text { a3 } \times 0.8 \text { (efficacy } \\
\text { from KZN trial, } \\
\text { hypothetical coverage) }\end{array}$ & Coverage $80 \%$ and $95 \%$ \\
\hline e2 & Net cost (= numerator for ICER) & $\$ 2764$ & $\mathrm{~d} 4-\mathrm{c} 4$ & \\
\hline e3 & ICER & $1 \cdot 23$ & e2/e1 & \\
\hline
\end{tabular}

VA, universal vitamin A supplementation alone; VAZ, universal Zn plus vitamin A supplementation.

${ }^{*}$ All costs are in international dollars adjusted to base year 2004.

Table 4 Incremental cost-effectiveness ratios (ICER) at varying prevalence of stunting with other base case parameters kept constant*

\begin{tabular}{llll}
\hline Prevalence of stunting (\%) & Total cost of VAt & Total cost of VAZ† & ICER $\ddagger$ \\
\hline 5 & $\$ 52565$ & $\$ 69993$ & $\$ 23 \cdot 18$ per additional case prevented by VAZ \\
10 & $\$ 67853$ & $\$ 77949$ & $\$ 6 \cdot 71$ per additional case prevented by VAZ \\
15 & $\$ 83141$ & $\$ 85905$ & $\$ 1 \cdot 23$ per additional case prevented by VAZ \\
20 & $\$ 98429$ & $\$ 93861$ & Negative net cost $(-1 \cdot 52)$ means VAZ is cost-saving relative to VA \\
25 & $\$ 113717$ & $\$ 101817$ & Negative net cost $(-3 \cdot 16)$ means VAZ is cost-saving relative to VA
\end{tabular}

VA, universal vitamin A supplementation alone; VAZ, universal Zn plus vitamin A supplementation.

${ }^{*}$ All costs are in international dollars adjusted to base year 2004.

†Total cost includes cost of intervention at $80 \%$ coverage, plus cost of diarrhoeal illness in stunted children at $\$$ Int $7 \cdot 80 /$ episode.

$\ddagger$ ICER calculated as net cost per episode of diarrhoea prevented, assuming that additional Zn supplementation benefits only stunted children. Where net cost is a negative value it means that VAZ is dominant; that is, it is cost-saving relative to VA. In that case a descriptive summary of ICER is provided rather than a cost per episode prevented. 
would result in a greater number of diarrhoea episodes prevented and hence total cost of illness prevented. At 95\% coverage VAZ showed low ICER at 25\% prevalence of stunting, but became cost-saving at a higher prevalence. If we assume that the preventive effect of VAZ is not restricted to stunted children but has a smaller effect among non-stunted children, then fairly low ICER is achieved at stunting prevalence between 10 and 15\%, with cost savings by $20 \%$. Doubling the cost of VAZ resulted in low ICER at $25 \%$ prevalence of stunting. A scenario of $5 \%$ HIV prevalence showed low ICER at $15 \%$ prevalence of stunting and cost saving by $20 \%$ prevalence. Results were sensitive to cost of diarrhoea episodes and cost and coverage of the intervention (Table 5). Generally, VAZ showed low incremental costs in several sensitivity analyses when the prevalence of stunting was close to $20 \%$.

\section{Discussion}

Prevalence of stunting in children aged under 5 years, supported by $\mathrm{Zn}$ intake data, is an indicator for quantifying $\mathrm{Zn}$ deficiency in populations ${ }^{(23)}$. Zn trials in regions with moderate to high stunting prevalence may show overall benefits or subgroup effects among stunted children ${ }^{(24,25)}$. This suggests that routine $\mathrm{Zn}$ supplementation as a public health intervention would be most effective if targeted to high-risk groups. Individual-level targeting requires feasible case detection strategies that are affected by cost and technical issues such as measurement error and misclassification of stunting. WHO considers stunting prevalence rates of $\geq 20 \%$ to be of public health concern ${ }^{(26)}$. In South Africa the prevalence of stunting varies, with some provinces and rural areas reaching prevalence of $25 \%$ or more - in general reflecting high to intermediate risk of population $\mathrm{Zn}$ deficiency ${ }^{(27)}$. Of note is that $\mathrm{Zn}$ deficiency prevalence estimates from South African national surveys use data on stunting and $\mathrm{Zn}$ intake. In the setting of such information, we propose that a threshold analysis that incorporates stunting prevalence and functional outcomes would be useful to policy makers.

Using the cost of diarrhoeal illness as an outcome of public health importance, we show that by $20 \%$ prevalence of stunting, universal VAZ dominated VA for many scenarios. In other words, it became relatively costsaving because the additional costs of VAZ compared with VA were negated by the cost of illness prevented by VAZ. This was fairly robust to changes in efficacy but sensitive to high VAZ costs and high coverage needs. At stunting prevalence between 15 and 20\%, the ICER should be interpreted in the context of actual resources available and other competing interventions. For example, at $15 \%$ stunting prevalence, an incremental cost of $\$$ Int $1 \cdot 23$ per additional case of diarrhoea prevented (Table 4) could translate crudely to an increment of \$Int 18 per additional 
DALY averted if we assume: (i) a diarrhoea case fatality rate of 2/1000; (ii) that a death in infancy corresponds to 33 DALY (based on WHO DALY estimates); (iii) that duration of an illness is $7 \mathrm{~d}$; and (iv) that disability weighting for a diarrhoeal episode is $0 \cdot 12^{(28-30)}$. This would then be weighed against the DALY averted by other interventions, albeit not taking into account the longer-term impact of diarrhoea, specifically of recurrent diarrhoea on stunting and cognition. Below $15 \%$ prevalence universal VAZ was not advisable. Illness costs were very influential in determining cost-effectiveness, implying that low-cost measures that drastically reduce severity of episodes and avert complications could theoretically displace prophylactic $\mathrm{Zn}$ supplementation as a preferred intervention.

Limitations to our model are inherent in our key assumptions. We have not incorporated newer interventions like universal rotavirus immunization, which was introduced subsequent to our study. The impact of HIV may have also changed, with infants now commencing antiretroviral treatment at much earlier ages. It is very likely that we have underestimated the cost of diarrhoeal illness. At higher costs of illness however, VAZ would become even more cost-effective and the threshold stunting prevalence at which VA is dominated may be even lower. The model does not consider other potential effects of $\mathrm{Zn}$ supplementation, such as reduced acute respiratory infections, reduced all-cause mortality and potential reduction of stunting, which would strengthen cost-effectiveness ${ }^{(31)}$. Although the input parameters used were based on a South African trial and health service setting combined with international and regional data, the simulation's structure has applicability to various geographic regions. This can be achieved by using data that may be locally available or updated and retaining sensitivity analyses that are relevant to local or regional settings.

The largest challenge is the paucity of programmatic data for preventive $\mathrm{Zn}$ supplementation. We have opted to use costs based on micronutrient powders, which may need further adaptation to accommodate local delivery mechanisms. The adherence with daily micronutrients in a programmatic setting is undeniably crucial to success of the intervention. It is also very likely that the costs of VAZ are underestimated and would require higher operational costs for delivery; however, the current developments in primary health care in South Africa make the delivery mechanism more feasible. South Africa is in the process of strengthening community-based services with community health workers already hired and trained to conduct regular home visits. Growth monitoring visits at primary health facilities or within community sites are another potential delivery point because they are scheduled to occur monthly in infancy and every second month in the second year of life. The service infrastructure therefore can already start considering incorporation of micronutrient supplementation. A pilot site based on geographic prevalence of stunting could then be used to better determine programmatic logistics and costs. This approach will also necessitate periodic surveys (and costs thereof) to monitor population $\mathrm{Zn}$ status dissociated by urban/rural and/or other categories.

\section{Conclusion}

Where evidence for preventive supplementation under realistic programme conditions is sparse, simulation models, like the one presented here, are useful preliminary exercises to explore cost-effectiveness. Our findings suggest that universal VAZ could replace VA in diarrhoea prevention programmes in South Africa using geographic targeting based on prevalence of stunting. This simulation needs ongoing validation under programmatic conditions where nutrition programmes are integrated with other child health programmes and through incorporation of more extensive programmatic cost data.

\section{Acknowledgements}

Sources of funding: This work was supported by grants from the US National Institutes of Health (1 UO1 AI45508-01, $1 \mathrm{~K} 24 \mathrm{AI} / \mathrm{HDO} 1671-01$ and D43TW05572-01), the Eunice Kennedy Shriver National Institute of Child Health and Human Development (NICHD)/Fogarty International Center (FIC) International Maternal and Child Health Training Grant (D43-TW001277-11 to M.L.B.) and the Wellcome Trust (grants 62925 to M.L.B. and 063009 to J.V.d.B.); and an International Nutrition Foundation Fellowship to M.K.C. Conflicts of interest: There are no conflicts of interest to declare. Authors' contributions: M.K.C. designed and conducted the current analysis and prepared the initial manuscript draft. M.L.B. was the principal investigator and responsible for study design of the micronutrient trial. J.V.d.B. was project director. K.-K.A.L. was project leader and study physician. N.M. supervised field activities and field data coordination. The manuscript was critically reviewed and approved by all of the authors. Acknowledgements: The authors acknowledge the following institution that permitted access to data used in this analysis: the Health Economics and HIV/AIDS Research Division (HEARD) of the University of KwaZulu-Natal, Durban, South Africa. They would also like to acknowledge the following individuals from Tufts University for review of and advice in preparing the manuscript: Drs Katherine Tucker, Jeffrey Griffiths, Parke Wilde and Joshua Cohen.

\section{References}

1. Statistics South Africa (2010) Mortality and Causes of Death in South Africa, 2008: Findings from Death Notification. Pretoria: Statistics South Africa; available at http:// www.statssa.gov.za/publications/P03093/P030932008.pdf 
2. Lorntz B, Soares AM, Moore SR et al. (2006) Early childhood diarrhea predicts impaired school performance. Pediatr Infect Dis I 25, 513-520.

3. Brown KH, Peerson JM, Baker SK et al. (2009) Preventive zinc supplementation among infants, preschoolers, and older prepubertal children. Food Nutr Bull 30, 1 Suppl., S12-S40.

4. World Health Organization (2005) Technical Updates of the Guidelines on Integrated Management of Childhood Illness (IMCI): Evidence and Recommendations for Further Adaptations. Geneva: WHO.

5. Edejer TT, Aikins M, Black R et al. (2005) Cost effectiveness analysis of strategies for child health in developing countries. BMJ 331, 1177.

6. International Zinc Nutrition Consultative Group (2007) Quantifying the Risk of Zinc Deficiency: Recommended Indicators. IZiNCG Technical Brief no. 1. Davis, CA: IZiNCG; available at http://www.izincg.org/Media/Default/ Publications/Files/English_brief1.pdf

7. Chhagan MK, Van den Broeck J, Luabeya KK et al. (2009) Effect of micronutrient supplementation on diarrhoeal disease among stunted children in rural South Africa. Eur J Clin Nutr 63, 850-857.

8. Umeta M, West CE, Haidar J et al. (2000) Zinc supplementation and stunted infants in Ethiopia: a randomised controlled trial. Lancet 355, 2021-2026.

9. Ching P, Birmingham M, Goodman T et al. (2000) Childhood mortality impact and costs of integrating vitamin $\mathrm{A}$ supplementation into immunization campaigns. Am J Public Health 90, 1526-1529.

10. Luabeya KK, Mpontshane N, Mackay M et al. (2007) Zinc or multiple micronutrient supplementation to reduce diarrhea and respiratory disease in South African children: a randomized controlled trial. PLOS ONE 2, e541.

11. Parikh A \& Veenstra N (2008) The evolving impact of HIV/AIDS on outpatient health services in KwaZulu-Natal, South Africa. S Afr Med J 98, 468-472.

12. Veenstra N \& Oyier A (2006) The burden of HIV-related illness on outpatient health services in KwaZulu-Natal, South Africa. AIDS Care 18, 262-268.

13. Adam T, Evans DB \& Murray CJ (2003) Econometric estimation of country-specific hospital costs. Cost Eff Resour Alloc 1, 3.

14. World Health Organization CHOosing Interventions that are Cost Effective (2006) Results of Unit Costs for patient services in the 14 GBD Regions. http://www.who.int/choice/ costs/unit_regions/en/index.html (accessed April 2006).

15. Statistics South Africa (2004) Discussion Paper. Consumer Price Index - Rural Areas and Total Country, January 2004. Pretoria: Statistics South Africa; available at http://www.statssa.gov.za/publications/DiscussCPIRural/ DiscussCPIRuralJanuary2004.pdf

16. Evans DB, Edejer TT, Adam T et al. (2005) Methods to assess the costs and health effects of interventions for improving health in developing countries. BMJ 331, 1137-1140.
17. Olsen JA \& Richardson J (1999) Production gains from health care: what should be included in cost-effectiveness analyses? Soc Sci Med 49, 17-26.

18. Horton S, Shekar M, McDonald C et al. (2010) Scaling Up Nutrition. What Will It Cost? Washington, DC: The World Bank

19. Bobat R, Coovadia H, Stephen C et al. (2005) Safety and efficacy of zinc supplementation for children with HIV-1 infection in South Africa: a randomised double-blind placebo-controlled trial. Lancet 366, 1862-1867.

20. Fischer TK, Anh DD, Antil L et al. (2005) Health care costs of diarrheal disease and estimates of the cost-effectiveness of rotavirus vaccination in Vietnam. I Infect Dis 192, 1720-1726.

21. Commission on Macroeconomics and Health (2001) Macroeconomics and Health: Investing in Health for Economic Development. Boston, MA: Center for International Development at Harvard University.

22. World Health Organization (2002) World Health Report 2002: Reducing Risks, Promoting Healthy Life. Geneva: WHO.

23. Hess SY, Peerson JM, King JC et al. (2007) Use of serum zinc concentration as an indicator of population zinc status. Food Nutr Bull 28, 3 Suppl., S403-S429.

24. Bhutta ZA, Bird SM, Black RE et al. (2000) Therapeutic effects of oral zinc in acute and persistent diarrhea in children in developing countries: pooled analysis of randomized controlled trials. Am J Clin Nutr 72, 1516-1522.

25. Rahman MM, Vermund SH, Wahed MA et al. (2001) Simultaneous zinc and vitamin A supplementation in Bangladeshi children: randomised double blind controlled trial. BMJ 323, 314-318.

26. International Zinc Nutrition Consultative Group, Brown KH, Rivera JA et al. (2004) International Zinc Nutrition Consultative Group (IZiNCG) technical document \#1. Assessment of the risk of zinc deficiency in populations and options for its control. Food Nutr Bull 25, 1 Suppl. 2, S130-S162.

27. Labadarios D, Steyn NP, Maunder E et al. (2005) The National Food Consumption Survey (NFCS): South Africa, 1999. Public Health Nutr 8, 533-543.

28. Mathers CD, Vos T, Lopez AD et al. (2001) National Burden of Disease Studies: A Practical Guide, Edition 2-0. Geneva: WHO, Global Program on Evidence for Health Policy.

29. World Health Organization (2004) The Global Burden of Disease: 2004 Update. Geneva: WHO.

30. Barungi M \& Kasirye I (2011) Cost-Effectiveness of Water Interventions: The Case for Public-Stand Pipes and BoreHoles in Reducing Diarrbea Among Urban Children in Uganda. Kampala: Economic Policy Research Centre, Makerere University.

31. Chhagan MK, Van den Broeck J, Luabeya KK et al. (2010) Effect on longitudinal growth and anemia of zinc or multiple micronutrients added to vitamin A: a randomized controlled trial in children aged 6-24 months. BMC Public Health 10, 145. 